Available online at GSC Online Press Directory

GSC Biological and Pharmaceutical Sciences

e-ISSN: 2581-3250, CODEN (USA): GBPSC2

Journal homepage: https://www.gsconlinepress.com/journals/gscbps

(REVIEW ARTICLE)

\title{
Optimization of management of patients with chronic heart failure taking into account cardiovascular functional status
}

\author{
Muyassar Gafurdjanovna Mukhamedova ${ }^{1,2, *}$ and Dildora Saidjanovna Narzullaeva ${ }^{3}$ \\ ${ }^{1}$ Cardiology department of Tashkent Postgraduate Medical Institute, Uzbekistan. \\ ${ }^{2}$ Cardiology department of Clinic-Pro JSC, Tashkent, Uzbekistan. \\ ${ }^{3}$ Internal Medicine department of Bukhara State Medical Institute, Bukhara, Uzbekistan.
}

Publication history: Received on 12 August 2020; revised on 23 August 2020; accepted on 26 August 2020

Article DOI: https://doi.org/10.30574/gscbps.2020.12.2.0263

\begin{abstract}
This review article summarizes the reasons, pathophysiological mechanisms, possible clinical features, and the optimal management strategies of patients with chronic heart failure taking into account cardiovascular functional status. In addition, it shows current situation with chronic heart failure in the world, epidemiological status of the problem as well, risk factors contributing to the development of the disease.
\end{abstract}

Keywords: Chronic heart failure; Ischemic heart disease; Cardiovascular status

\section{Introduction}

Chronic heart failure (CHF) is becoming one of the leading problems in modern medicine and is widespread; extremely unfavorable: forecast and high financial costs [1]. Despite the successes achieved in medical and surgical methods of treatment, chronic heart failure (CHF) is still one of the most common, progressive and prognostically unfavorable diseases of the cardiovascular system [2]. It is known that the proportion of heart failure in the structure of cardiovascular diseases (CVD) is on average $40.0-45.0 \%$ [3].

The main task of identifying and treating patients with heart failure lies with the doctors of the outpatient service, since it is known that almost $80 \%$ of the population begins and ends with examination and treatment in primary health care, and about $83 \%$ of patients with heart failure are treated not by cardiologists, but by doctors primary link [4]. However, CHF is still not included in the list of cardiovascular diseases subject to active follow-up.

According to the study "EPOHA-O-CHF" (2002), the purpose of which was to identify patients with heart failure by referring to medical institutions, it turned out that every third patient who comes to the clinic or hospital has signs of heart failure [5]. According to the epidemiological study "EPOCH-O-CHF" conducted in 8 regions of the European part of Russia among the unorganized population, among patients with CHF, patients with initial stages and a functional class (FC) of CHF prevail: the proportion of people with "mild" CHF (I-II FC according to classification The New York Heart Association (NYHA)) is 4 times more - 9.8\% than with "severe" (III - IV FC) - 2.5\% [6]. Moreover, 84.8\% of all patients with "mild" CHF and 37.4\% with "severe" seek help at the clinic [7]. Domestic study "EPOCHA" showed that in our country the main medical efforts are not aimed at outpatient treatment of the initial stages of heart failure and the prevention of its progression and complications, but towards ineffective inpatient treatment of terminal stages [8]. A.L.Alyavi et al. [9] believes that an increase in the role of the outpatient unit in the early diagnosis and treatment of outpatients with heart failure will help improve the situation with heart failure.

\footnotetext{
${ }^{*}$ Corresponding author: Muyassar Gafurdjanovna Mukhamedova

Cardiology department of Tashkent Postgraduate Medical Institute, Uzbekistan.
} 
In addition, the proportion of those patients in whom CHF develops with a preserved ejection fraction (EF) of the left ventricle (LV) (EF <40\%) is constantly increasing. So, according to the EPOHA-O-CHF study (2002), the proportion of patients with preserved PV exceeded $80 \%$ for the outpatient cohort [10]. These are cases of diastolic heart failure, which has flow patterns and difficulties in therapy [11].

Recent studies have shown that the work of general practitioners (GPs), or family doctors, is a more effective and economical form of primary health care [12]. However, the problems of diagnosis and management of patients with heart failure among GPs have not yet been studied.

Single studies of foreign and domestic authors have proved that timely diagnosis of heart failure, including clinical heart failure, causes difficulties for primary care doctors, since there are still not enough sensitive and specific subjective criteria for this condition [13]. Therefore, objective evidence of systolic or diastolic LV dysfunctions is required to confirm CHF. In clinical practice, echocardiography (Echocardiography) is the most accessible for determining these functions. However, according to foreign studies, echocardiography is used by doctors when diagnosing heart failure from 36 to $49 \%$ of cases [14]; in domestic - in $69.7 \%$ of outpatients with heart failure [15]. Therefore, there is a problem of hypo- and overdiagnosis of heart failure [16].

Heart failure" in 2012 there were ESC recommendations for the diagnosis and treatment of heart failure, but the gap between the achievements of modern cardiology, clinical pharmacology and the use of this knowledge in practical medicine is still quite large. Thus, the awareness of primary care physicians about heart failure, as shown by the results of IMPROVEMENT (2000) and SHAPE (2003), is insufficient. And, as a result of this, according to the results of the "EPOHA-O-CHF" study (2002) in Russia, patients with CHF are not "healed" by the main groups of drugs [16].

In addition, the effectiveness of the treatment of heart failure largely depends on how carefully the patient complies with the doctor's recommendations regarding medication and lifestyle changes [17]. Studies of foreign authors have shown that the most common cause of decompensation of heart failure is failure to comply with the recommendations of the attending physician [18]. In Russia, studies that are devoted to the study of adherence to the treatment of patients with heart failure are rare [19]. In addition, the level of self-monitoring of patients with heart failure has not been studied in detail by domestic authors.

Currently, new forms of management and treatment of patients with heart failure have appeared, one of which is structured therapeutic education of patients. Training programs in different regions of our country have differences (forms of training, intensity, number of topics used for training patients with heart failure), and there is still no single program. Most studies on the effectiveness of training have been conducted with patients who, as a rule, have III - IV FC according to NYHA, mainly in large cardiology centers and hospitals. They confirmed that patient education leads to a decrease in the number of repeated hospitalizations, cost savings, a tendency to a decrease in mortality is noted, which made it possible to think about the advisability of introducing the education of patients with heart failure in the conditions of GP work.

The results of the famous Framingham study showed that in the United States in the population of people over 45 years of age, the number of patients with clinically severe heart failure is approximately $2.5 \%$, or 5 million people, in absolute numbers. Every year, this "army" is replenished with another 400,000 patients. In the first chapter of the Recommendations of the European Society of Cardiology for the Diagnosis and Treatment of Heart Failure, published in September 2001; G., indicated, that, the prevalence of symptomatic heart failure in the European population ranges from 0.4 to $2.2 \%$. According to research; held since the $70 \mathrm{~s}$; the prevalence of heart failure in the United States and Western Europe is on average from 3 to 20 people per 1000 population and this indicator has been steadily increasing over the course of 30 years [20].

The concept of an impending epidemic: CHF was "calculated" on a simulation model of the development of the disease: by 2010 on the example of the Netherlands (Woppeich L. e1 a1., 1994). In absolute terms, an increase in CHF prevalence of $70 \%$ is predicted. This growth will clearly increase as the population ages [21].

The general "aging" of the population [22], the improvement of early diagnosis (the appearance of ultrasound in> cardiology) and the improvement of therapeutic and surgical methods of treatment, mainly IHD, as well as dilated cardiomyopathy and heart defects, are an objective reason for the increase in the prevalence of cardiac insufficiency. One must be prepared for the fact that every 10 or 3 patients after visiting a cardiologist (or therapist) will leave his office with this diagnosis in 10-20 years [23]. 
In addition to widespread, heart failure is characterized by a high level of disability, mortality. Suffice it to say that $70 \%$ of men and $63 \%$ of women diagnosed with CHF die within a few years after the first clinical signs of the disease appear, and up to half of all deaths occur in the first year of the disease [24]. It is paradoxical that the prognosis of patients with heart failure is often worse than that of many cancer patients [25]. According to the data of an epidemiological study completed in $<2003$ in the Russian Federation, EP0XA-XGH, the prevalence of clinically expressed CHF in the Uzbekistan population is 5.5\%, which is 3-10 times higher than in the West. If we take into account patients with asymptomatic left ventricular dysfunction, then we can talk about $11.7 \%$ of the population, or about 16 million people [26]. According to the latest updated data, the prevalence of CHF in the European part of the Russian Federation in accordance with criteria corresponding to CHF I-IV FC was $12.3 \%$ [27]. The study showed that poorly treated arterial hypertension (AH) is the main cause of heart failure in Russia, as in Europe.

Patients with cardiovascular disease and concomitant heart failure (HF) are extremely difficult to manage in the category of patients. One of the main problems that a doctor encounters in treating such a patient is a high risk of thrombotic complications of various localization, associated with both the underlying disease (atherosclerosis, arterial hypertension) and the presence of heart failure [28].

Today, there is no doubt that people with diagnosed diseases of the cardiovascular system (coronary heart disease (CHD), cerebral and peripheral arteriosclerosis, arterial hypertension) belong to the category of patients with a high and very high risk of acute vascular events $[12,4]$. However, one should not forget that heart failure is also a proven independent predictor of acute vascular events in both cardiac and cerebral localization [10]. In particular, it was found that a decrease in the ejection fraction of the left ventricle (LVEF) for every $5 \%$ is accompanied by an increase in the risk of thrombotic complications by $18 \%$.

It should be noted that patients with chronic heart failure (CHF) have a high risk of developing acute vascular events, regardless of the primary pathological process, since the absence of an adequate LV systole leads to a total slowdown in blood flow, sludge and blood stasis in microvessels, local and systemic activation of thrombosis, an increase in blood viscosity, the progression of endothelial dysfunction. As a result of this, the likelihood of developing both local ischemic events and systemic thromboembolic complications significantly increases [29].

Considering that a prolonged course of CHF leads to a significant depletion of reserve abilities of the endothelium as the main factor in maintaining the stability of hemovascular homeostasis that impedes the development of parietal thrombosis, effective stabilization of endothelial-platelet interaction is of particular importance for the implementation of antithrombotic prophylaxis [30].

Accordingly, the conduct of antithrombotic prophylaxis in CHF should include both antiplatelet and endothelioprotective drugs [31]. It should be remembered that the stimulation of eNOS to activate the synthesis of nitric oxide (NO) in patients with heart failure may be unsuccessful due to the long course of the pathological process, depleting the reserves of the substrate for the synthesis of NO L-arginine, activation of local inflammation and free radical oxidation, and the whole a number of other reasons leading to a change in the structure of eNOS [32].

In this regard, for the correction of endothelial dysfunction in patients with heart failure, along with standard drugs with a pleiotropic endothelioprotective effect (ACE inhibitors, beta-blockers with additional properties, statins), it is necessary to use drugs that replenish the deficiency of the precursor NO L-arginine, the reserves of which are conditions of prolonged (including pharmacological) stimulation of the endothelium and impaired amino acid resynthesis in the liver and distal tubules of the kidneys are depleted.

Studies have shown that periodic administration of L-arginine in patients with heart failure helps to improve the functional state of the endothelium, increase the duration of the test with 6 minutes of walking, improve the quality of life of patients, in addition, data were obtained on the positive effect of this amino acid on central hemodynamics, inflammatory activity processes and peroxidation processes [33].

Also, in order to "facilitate" the work of eNOS, drugs activating the release of prostacyclin, an alternative endothelial vasodilator, should be used. Triflusal, which, unlike acetylsalicylic acid (ASA), increases the synthesis of prostacyclin by endothelium, can be recommended as such a drug, while it has the additional ability (by increasing the level of cGMP) to increase the synthesis of endothelium NO and also has a double antiplatelet effect: it selectively blocks platelet cyclooxygenase-1 (COX-1), which disrupts the synthesis of thromboxane and reduces the activity of phosphodiesterase, as a result of which calcium-dependent platelet aggregation is blocked [34]. 


\section{Conclusion}

Thus, the subpopulation of patients with chronic heart failure more advanced FC against the background of IHD is characterized by severe clinical, paraclinical and morphofunctional disorders. The quality of life of patients is reduced, which is mainly due to manifest manifestations of pathology and limitations of physical activity.

\section{Compliance with ethical standards}

\section{Acknowledgments}

Authors thank to the M. G. Mukhamedova for the design and critical review of the manuscript.

\section{Disclosure of conflict of interest}

None

\section{References}

[1] Alyavi A and Uzokov J. (2017). Treatment of stable angina pectoris: focus on the role of calcium antagonists and ACE inhibitors. Ont Health Technol Assess Ser, 15(9), 1-12.

[2] Payziev D, Azizov SI, Uzokov JK and Iskhakov SA. (2020). P1003 New onset atrial fibrillation after stenting of the right coronary artery in patients with ischemic disease of the heart. EP Europace, 22(S1), 162-338.

[3] Cabrera CC, Ekström M, Linde C, Persson H, Hage C, Eriksson MJ and Lyngå P. (2020). Increased iron absorption in patients with chronic heart failure and iron deficiency: Iron absorption in heart failure. J Card Fail. In press.

[4] Radjabova DI, et al. (2018). The Features of Cytokine Status in Patients with Coronary Heart Disease. Hypertension \& Vasc Biol Int J, 1(1), 000101.

[5] Mamatkulov, KhA, Usarov MH, Uzokov ZhK, Arzieva, JB. (2015). Influence of interventional myocardial revascularization in patients with acute myocardial infarction without " $q$ " wave. 0 'zbekiston terapiya axborotnomasi, 2, 18.

[6] Uzokov JK, Alyavi BA and Abdullaev AX. (2019). Assessment of the clopidogrel action with regard to CYP2C19 gene polymorphisms in patients with coronary artery disease after implantation of des stents. Euroasian Card J, S2, 309-309.

[7] Belfiore A, Palmieri VO, Di Gennaro C, Settimo E, De Sario MG, Lattanzio S and Portincasa P. (2020). Long-term management of chronic heart failure patients in internal medicine. Internal and Emer Med, 15(1), 49-58.

[8] Alyavi B, Uzokov JK, Abdullaev AX and Payziev DD. (2020). P427 Influence of ranolazine on cardioversion rate in patients with new onset atrial fibrillation and ischemic disease of the heart. EP Europace, 22(S1), 162-337.

[9] Abdullaev AH, Alyavi BA, Iskhakov SHA, Uzakov ZhK, Ibabekova ShR, Yunusova LI, Azizov ShI. (2018). Some indicators of biochemical studies and echocardiography of patients with coronary heart disease who underwent stenting. In VI Eurasian Congress of Cardiology, 76.

[10] Habib G, Lancellotti P, Erba PA, Sadeghpour A, Meshaal M, Sambola A and Cosyns B. (2019). The ESC-EORP EUROENDO (European Infective Endocarditis) registry. Eur Heart J-Qual C Clin Out, 5(3), 202-207.

[11] Alyavi BA et al. (2018). Clinical case of endovascular treatment of chronic cerebral arterial insufficiency in a patient with critical ischemia of the lower extremities. Bukovynskiy medical visnik, 22 (2), 86.

[12] Abdullaev AKh et al. (2018). Some approaches to improve the effectiveness of the treatment of coronary heart disease. VI Eurasian Congress of Cardiology, 75-76.

[13] Edelmann F, Knosalla C, Mörike K, Muth C, Prien P, Störk S and Group HFD. (2018). Chronic heart failure. Deut Ärz Inter, 115(8), 124.

[14] Alyavi A, et al. (2018). Influence of Diet with Low Glycemic Index on Lipid Spectrum in Patients with Ischemic Disease of the Heart. Atherosclerosis Supplements, (32), 158.

[15] Radjabova DI, Alyavi AL, Alyavi BA, Tulyaganova DK, Uzokov JK, Shodiev JD and Nuritdinova SK. (2018). The Features of Cytokine Status in Patients with Coronary Heart. 
[16] Alyavi BA et al. (2019). On an integrated approach to the treatment and rehabilitation of patients with ischemic heart disease. Eurasian Journal of Cardiology, S2, 154-154.

[17] Abdullaev AH et al. (2019). Evaluation of the effectiveness of metabolic therapy in the complex treatment of coronary heart disease. Eurasian Journal of Cardiology, S2, 147-148.

[18] Alyavi BA et al. (2019). The functional state of the liver in patients with ischemic heart disease. Eurasian Journal of Cardiology, S2, 155-155.

[19] Uzokov J. (2020). Influence of abnormal lipid components in statin-naive young adults: Is there any gap?. Eur J Prev Card, 27(8), 868-869.

[20] Lyutfullayevich AA, et al. (2017). Relationship between hemodynamic parametres and NPPA, NPPB, NPR3 genes polymorphism in patients with ischemic heart disease. International scientific review, 7(38).

[21] Dick SA and Epelman S. (2018). Chronic heart failure and inflammation: what do we really know?. Circ Res, $119(1), 159-176$.

[22] Park JB. (2016). The Pulse of Asia 2016 Seoul, September 24-26, 2016, Seoul, Republic of Korea: Abstracts. Pulse, $4(2-3), 93$.

[23] Alyavi AL, et al. (2017).Predictors of contrast induced nephropathy in patients with metabolic syndrome. Eur Heart J, 38, 1283-1283.

[24] Babaev M, et al. (2018). Influence of l-arginine aspartate on vascular markers in hypertensive patients with metabolic syndrome. Eur Heart J, 39, 495-495.

[25] Alyavi BA et al. (2019). Influence of lipid-lowering and antiplatelet therapy on some biochemical and ultrasound parameters of patients with ischemic heart disease who underwent stenting. Cardiovascular Therapy and Prevention, 18 (S1), 12-13.

[26] Abdullaev AH et al. (2019). Evaluation of the effectiveness of colstres in patients with coronary heart disease. Cardiovascular Therapy and Prevention, 18 (S1), 5-6.

[27] Iskhakov S, et al. (2019). Comparative Analysis of The Inflammatory Biomarkers In Patients With Stable Coronary Artery Disease And Metabolic Syndrome. Atherosclerosis, 287, e171.

[28] Lutfullayevich AA, et al. (2017). GW28-e0698 Telmisartan with amlodipine versus lisinopril with amlodipine on home blood pressure variability in patients with metabolic syndrome. J Amer Coll Card, 70(16), 138.

[29] Sidalai RC and dr. (2015). $\beta$-adrenoblocators and myocardial infarction myocardial infarction. External Medicine Examiner, 2.

[30] Usarov M, et al. (2016). Efficacy of Combination Therapy Using Nebivalol and Trimetazidine In Hypertensive Patients With Metabolic Syndrome And Stable Angina. J Hyperten, 34, e349.

[31] Lutfullayevich AA, et al. (2017). Cardiovascular risk stratification and gender differences in hypertensive patients with metabolic syndrome. J Amer Col Card, 70(16), 138-139.

[32] Mukhamedova M, et al. (2019). P120 Relationship between left ventricular global function index and cardiac systolic functions in patients with chronic ischemic disease of the heart and diabetes mellitus. Eur Heart J-Card Imag, 20(S3), 147, 008.

[33] Ahmedov I, et al. (2016). Features of hyperreninemic aldosteronism in hypertension patients with metabolic syndrome. Eur J Prev Card, 23(1).

[34] Habib G, Erba PA, Iung B, Donal E, Cosyns B, Laroche C and Oliver L. (2019). Clinical presentation, aetiology and outcome of infective endocarditis. Results of the ESC-EORP EURO-ENDO (European infective endocarditis) registry: a prospective cohort study. Eur Heart J, 40(39), 3222-3232. 